Article

\title{
Investigation of a Passive Sensor Array for Diagnosis of Loosening of Endoprosthetic Implants
}

\author{
Cathérine Ruther $^{1, *}$, Christian Schulze ${ }^{1}$, Andrea Boehme ${ }^{1}$, Hannes Nierath ${ }^{2}$, \\ Hartmut Ewald $^{2}$, Wolfram Mittelmeier ${ }^{1}$, Rainer Bader ${ }^{1}$ and Daniel Kluess ${ }^{1}$
}

1 Department of Orthopaedics, University Medicine Rostock, Doberaner Str. 142, 18057 Rostock, Germany; E-Mails: christian.schulze2@uni-rostock.de (C.S.);

andrea.boehme@uni-rostock.de (A.B.); wolfram.mittelmeier@med.uni-rostock.de (W.M.); rainer.bader@med.uni-rostock.de (R.B.); daniel.kluess@med.uni-rostock.de (D.K.)

2 Institute of General Electrical Engineering, University of Rostock, Albert-Einstein-Str. 2, 18055 Rostock, Germany; E-Mails: hannes.nierath@uni-rostock.de (H.N.); hartmut.ewald@uni-rostock.de (H.E.)

* Author to whom correspondence should be addressed; E-Mail: catherine.ruther@med.uni-rostock.de; Tel.: +49-381-4949-375; Fax: +49-381-4949-308.

Received: 23 October 2012; in revised form: 5 December 2012 / Accepted: 13 December 2012 / Published: 20 December 2012

\begin{abstract}
Currently, imaging methods are used to diagnose loosening of endoprosthetic implants, but fail to achieve $100 \%$ accuracy. In this study, a passive sensor array which is based on the interaction between magnetic oscillators inside the implant and an excitation coil outside the patient was investigated. The excited oscillators produce sound in the audible range, which varies according to the extent of loosening. By performing several experimental tests, the sensor array was optimized to guarantee reproducible and selective excitation of the sound emission. Variation in the distance between the oscillators demonstrated a definite influence on the quality of the generated sound signal. Furthermore, a numerical design analysis using the boundary element method was generated for consideration of the magnetic field and the selectivity of the oscillators during excitation. The numerical simulation of the coil showed the higher selectivity of a coil with a C-shape compared to a cylindrical coil. Based on these investigations, the passive sensor system reveals the potential for detection of implant loosening. Future aims include the further miniaturization of the oscillators and measurements to determine the sensitivity of the proposed sensor system.
\end{abstract}


Keywords: total joint replacement; aseptic loosening; intelligent implant; boundary element method; magnetic induction; loosening sensor

\section{Introduction}

Implantation of a total hip replacement (THR) has become a standard procedure for the treatment of degenerated or fractured hip joints. After resection of the femoral head, a stem is impacted or cemented into the femur. The acetabulum is reamed and a cup is placed into the cavity. The artificial joint is realized by connecting a ball-head to the stem and a liner into the cup. The modular design of modern total hip replacements allows choice of different materials as bearing surfaces in combination with materials of high biocompatibility and enhanced bone ingrowth. Despite more than forty years of clinical experience, complications can still occur which require an exchange (revision) of the THR or some of its components. The main reason for failure is loosening of the stem or the cup [1]. Acute pain requires clarification as to whether the THR is mechanically loose and has to be revised [2]. In orthopedic surgery, each revision of a THR leads to subsequent tissue defects and functional deficits. The sooner the loosening is detected, the better the prognosis for the new implant. However, clinically applied methods of assessing implant fixation with respect to implant loosening are of suboptimal precision, leading to uncertain indication of revision surgery and late recognition of bone defects. In clinical practice, imaging methods, especially radiography, are used to identify the actual condition of fixation, but fail in the aim of achieving $100 \%$ accuracy in the diagnosis of slight loosening $[3,4]$. Thus, vibration techniques were investigated experimentally to assess the structural integrity of endoprosthetic implant systems by evaluating acoustic and structural vibration parameters e.g., damping and resonant frequency [5-12]. Different research studies, including animal experiments, demonstrated a positive correlation between a decrease in the resonant frequency and a decrease of the system stiffness, and therefore an increase in implant loosening [13-16]. The focus of past studies was to obtain vibrational output by exciting the bone-implant-system using an external electrodynamic shaker at the femoral condyles [17-19]. The output signal could be measured with an accelerometer attached to the skin at the prominence of the greater trochanter. Resonant frequencies in the highly sensitive band starting at $2,500 \mathrm{~Hz}$ are most sensitive to changes in the biological structure and thus the condition of fixation of the THR [20]. This frequency range is difficult to reach using the shaker, since higher frequencies are reported to provoke pain. Currently, researchers are striving to replace ex vivo techniques with novel in vivo sensors, such as accelerometers in vibration analysis [21]. For any measurement system that uses electronics integrated in the THR, loss of functionality and degradation during sterilization presents a significant challenge. Besides miniaturized design and biocompatibility, a reliable power supply is an issue in development of in vivo sensors. The technology of choice for vibrometry in most studies is wireless powering and data telemetry. Hence, energy can be provided extracorporeally by a primary coil and an in vivo secondary coil integrated in the endoprosthetic implant. In order to ensure long-term performance of the in vivo electronics, inductivities and capacities have to be chosen carefully. During the design of application specific electric circuits, low operating voltages and extremely low input signal levels appear, resulting in offset voltages and 
crosstalk from digital to analogue wires [22]. A study with an artificial thigh and a THR equipped with an integrated accelerometer showed damping of frequencies in the highly sensitive band, even though an optimized amplification was applied [23].

Due to the aforementioned challenges of implementation of an integrated active system to identify implant loosening, we proposed a passive sensor concept based on magnetic induction [24]. The concept is characterized by an extracorporeal coil, which excites several magnetic oscillators inside the THR to impinge on thin membranes and thus producing a sound and vibration signal (Figure 1). The proposed passive concept consists of several spring-mass-oscillators, which are assembled by a flat spring and a permanent magnetic sphere. This kind of oscillating system can be excited using an excitation coil, due to the polarization of the sphere. The external magnetic field effects the displacement of the oscillator from its neutral position. Hence, the oscillator moves back and impinges on the membrane dependent on its back-spring force. From this central point, sound emission originates due to the excitation of a free oscillation of the membrane-bone system in its bending modes. The resultant structure-borne sound waves propagate as transient elastic sound waves through the adjacent tissue. At the border of the bone-membrane system, changes in the sound characteristics appear dependent on the condition of implant fixation.

Figure 1. Schematic illustration of the passive sensor array inside a total hip stem.

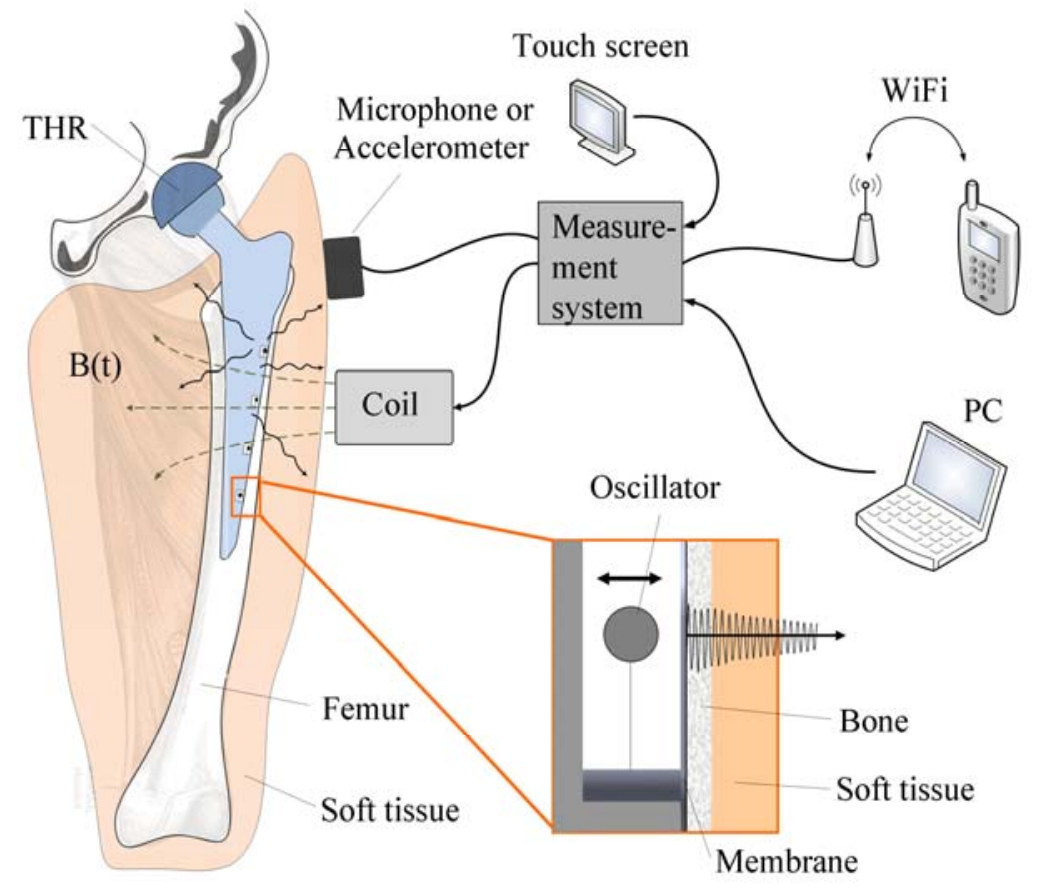

The sound emission and vibration of the system can be detected using a structure-borne sound sensor, e.g., an accelerometer or a microphone. To identify loosening at different locations of the hip stem, several integrated oscillators are necessary.

In the present work, the passive sensor array was investigated to guarantee the reproducible and selective excitation of sound emission inside the THR. In the study, one of the most important considerations is how to excite each individual oscillator without influencing oscillators proximal to the excited one, and therefore achieve the highest possible selectivity. In particular, we addressed the question of which parameter adjustments of the extracorporeal coil are most appropriate for the 
selective excitation of the oscillators. A numerical simulation of two different excitation coil designs, applying the boundary element method, was carried out.

\section{Methods}

\subsection{Parameter Analysis of the Internal System}

The distribution of magnetic field lines of a coil needs to be focussed in order to ensure a selective excitation of the oscillators. Undirected magnetic field lines lead to an activation of several oscillators simultaneously, hence influencing the selectivity of the excitation. The excitation of a single oscillator is of vital importance for avoiding sound interference, especially at one membrane, and hence ensuring highly sensitive measurements. There are different possibilities to achieve a selective excitation of the oscillators in order to measure the sound waves and vibration at each membrane. First, variation of the eigenfrequency of the oscillators ensures differentiation due to providing differently alternating voltages at the excitation coil. The flat spring of the oscillator can be represented as a beam with a constant stiffness and a point mass. By changing the geometry of the flat spring, the bending stiffness as a product of the Young's modulus E and the inertia I of the flat spring can be varied. Thus, the eigenfrequency of every single oscillator can be determined. Since the mass of the oscillator is not varied in the experimental tests, it can be neglected in further considerations. The bending of the beam can be described by the partial differential equation:

$$
\ddot{w}+\frac{E I}{\rho A} w=0
$$

The eigenfrequency $\omega_{\mathrm{K}}$ of the free oscillating beam in its different eigenmodes $\mathrm{k}$ arises from following equation:

$$
\omega_{K}=\left(k^{2} \pi^{2} L\right)^{2} \sqrt{\frac{E I}{\rho A L^{4}}}
$$

Based on this equation, different oscillators were chosen and tested in an experimental setup (Figure 2(a), Table 1). The oscillators were established by fixing a permanent magnetic sphere (Ø3 $\mathrm{mm}, \mathrm{NdFeB})$ on a flat steel spring with adhesive. The resonant frequency of each oscillator was measured using an M7L/4 distance laser sensor (MEL Mikroelektronik, Munich, Germany) and a cylindrical excitation coil, where a sweep signal of a function generator between 5 and $500 \mathrm{~Hz}$ was induced. The frequency with the highest amplitude equals the oscillators' eigenfrequency and was evaluated. Three oscillators, which had an acceptable difference in the eigenfrequency, were used for further investigations.

A further parameter that can be varied to achieve a selective excitation of the oscillator is the distance $d_{1}$ between the centre of the sphere and the membrane (Figure 2(b)). This is necessary, because an ideally chosen distance $d_{1}$ for the excited oscillator ensures the highest amplitude of membrane oscillations. A further reason is that due to the continuous excitation of the oscillator, the dynamic effects influence the energy of the back spring of the oscillator. Thus, a choice of distance $\mathrm{d}_{1}$ that is too small affects the proximate oscillator due to the induced energy of the coil. Hence, the proximate oscillator starts to impinge on the membrane, which should be avoided. Therefore, different distances $d_{1}$ were tested in an experimental setup (2.05, 2.10, 2.15, 2.20 and $\left.2.25 \mathrm{~mm}\right)$. 
Figure 2. Scheme of the experimental setup. (a) Measurement of the eigenfrequency of the oscillator. (b) Measurement of the displacement $\mathrm{d}_{2}$ of the oscillator after the first impingement, to find the ideal distance $d_{1}$ between the centre of the sphere and the membrane.

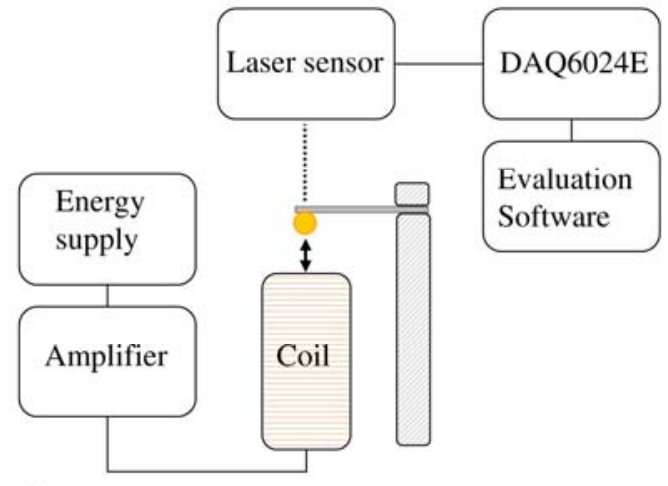

a.

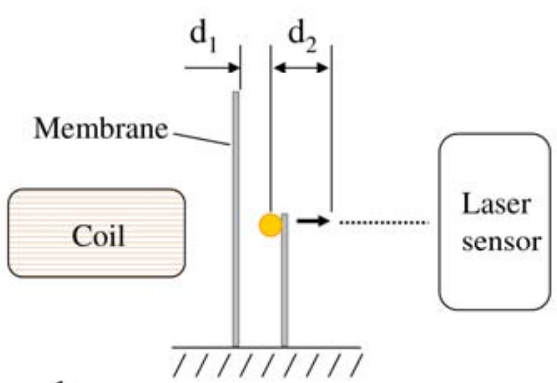

b.

Table 1. Geometries of the tested oscillators.

\begin{tabular}{|c|c|c|}
\hline Length $(\mathbf{m m})$ & Width $(\mathbf{m m})$ & Thickness $(\mathbf{m m})$ \\
\hline 3.00 & & \\
4.00 & & \\
5.00 & 2.00 and 1.00 & 0.05 \\
6.00 & each length & \\
7.00 & & \\
\hline 7.00 & 2.00 & 0.10 \\
\hline
\end{tabular}

The last parameter to configure the sensor array in order to achieve a selective excitation is the distance $\mathrm{d}_{3}$ between the oscillators (Figure 3 ). A high number of oscillators inside the total hip stem is desired to ensure the diagnosis over a wide surface. Therefore, the displacement $\mathrm{d}_{2}$ of the oscillator proximal to the excited oscillator was tested by fixing the two oscillators in a clamping device with different distances $\mathrm{d}_{3}(5,10,20,30,40$ and $60 \mathrm{~mm})$.

Figure 3. Experimental setup for investigation of the influence on the proximate oscillator during excitation of the desired oscillator by varying the distance $d_{3}$. (a) Schematic setup. (b) Top view of the experimental setup exemplarily shown for the distance $d_{3}=40 \mathrm{~mm}$, with the excitation of the oscillator on the left hand side.

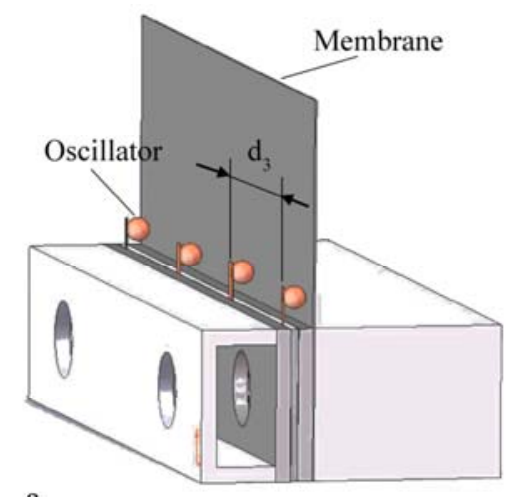

a.

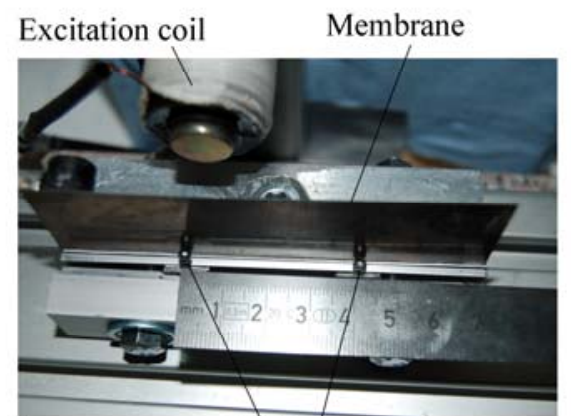

b.

Oscillator 
In order to realize a filigree module consisting of a membrane and oscillator as one component with a high long-term stability, generative procedures are necessary for the cost-effective manufacturing of the oscillators. Therefore, the geometries had to be adapted due to the application of biocompatible Ti6Al4V-alloy. This was done by using the resulting eigenfrequencies of the experiments for the best selectivity and hence varying the geometry of the flat spring until the desired eigenfrequency was achieved. The adaptation of the oscillator is simulated using the modal analysis tool of the Finite-Element-Software SIMULIA ABAQUS CAE Version 6.10 (Dassault Systems, Vélizy-Villacoublay, France). For the simulation, three different oscillator designs were established, based on the results of the experiments. The magnetic sphere was constructed as a point mass in the model. All parts were defined as deformable solids. The base of the membrane-oscillator system was fully encased. After a convergence analysis, 39,700 quadratic tetrahedron elements were used to mesh the parts. In order to calculate the eigenfrequency of the oscillators, the Lanczos solver was used.

\subsection{Design Analysis of the Extracorporeal System}

During the experiments to test the different oscillator types, we observed that the energy provided by the cylindrical coil over a distance of at least $100 \mathrm{~mm}$ would not be sufficient to achieve an excitation of the oscillators e.g., in a human leg. Using a cylindrical coil, the magnetic field lines cannot be concentrated, which is required for the selective displacement of the oscillators. Therefore, an inductive unit with a C-core for application at the patient's leg was designed using numerical simulation based on the boundary element method (FARADAY Version 6.3, Integrated Engineering Software, Winnipeg, MB, Canada). The current $\mathrm{I}_{\mathrm{ex}}$ and the number of windings $\mathrm{N}$ for an optimized excitation C-shape coil could be determined. In order to reduce the complexity of the model, the magnetic sphere can be transferred to the model of a conductor swing, which is positioned in the magnetic field with an imposed magnetic flux density $B_{\text {ex }}$ in y-direction (Figure 4). The permanent magnetic sphere provokes a magnetic flux density $B_{S}$ affecting $B_{\text {ex }}$. Both magnetic fields have an effect on the displacement force $F_{\text {dis }}$ that has to be produced to displace the oscillators. The conductor swing model is used, because the distribution of the magnetic field lines has its highest density in the center of the magnetic sphere. Furthermore, the development of the current $I$ at the surface along the equator perpendicular to the magnetic field lines was assumed.

Figure 4. Transfer of the magnetic sphere to a simplified conductor swing model for calculation of the magnetic flux density $B_{\text {ex }}$.

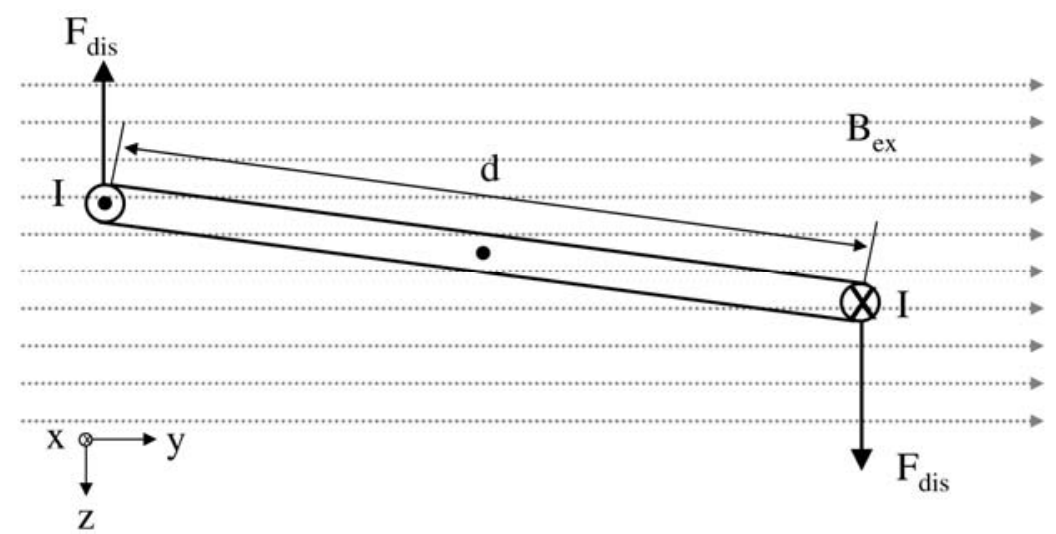


The magnetization $\mathrm{M}$ of the permanent magnetic sphere can be determined using the hysteresis curve. A simplification of the model is possible, if the magnetic field lines of $\mathrm{B}_{\mathrm{S}}$ are excluded. Without external field lines, the current I can be calculated using $M$ and the diameter $d$ of the magnetic sphere. This was realized by determining the current density $\mathrm{J}_{\mathrm{m}}$ in a first step:

$$
J_{m}=\operatorname{rot} M
$$

Following Stokes' thesis, the differential can be converted to an integral equation:

$$
\int_{A} J_{m} d A=\int_{A} \operatorname{rot} M d A=\oint_{A} M d l
$$

We can assume that the magnetization $M$ is constant over the small integration distance. Thus, $\mathrm{M}$ can be placed in front of the integral. As follows, $\mathrm{dl}$ can be replaced by the diameter $\mathrm{d}$ of the magnetic sphere:

$$
I=M d+\text { external field components }
$$

Analogously to the Lorentz force, $B_{\mathrm{ex}}$ is calculated. Therefore the force $\mathrm{F}_{\mathrm{dis}}$ acts on the live conductor, which operates in the external magnetic field. $F_{\text {dis }}$ of the oscillator could be calculated using the cross product.

$$
d \vec{F}=I(d \vec{l} \times \vec{B})
$$

In the next step, it was assumed that the current $\mathrm{I}, \mathrm{B}_{\mathrm{ex}}$ and $\mathrm{F}_{\mathrm{dis}}$ are linked over the right-hand rule. Current $\mathrm{I}$ in $\mathrm{x}$-direction is directed in the conductor swing on the right hand side. $\mathrm{B}_{\mathrm{ex}}$ affects the $\mathrm{y}$-direction and $\mathrm{F}_{\text {dis }}$ in $\mathrm{z}$-direction below:

$$
\left[\begin{array}{l}
d F_{\text {disx }} \\
d F_{\text {disy }} \\
d F_{\text {disz }}
\end{array}\right]=I\left(\left[\begin{array}{l}
d l_{x} \\
d l_{y} \\
d l_{z}
\end{array}\right] x\left[\begin{array}{l}
B_{e x x} \\
B_{e x y} \\
B_{e x z}
\end{array}\right]\right)
$$

After insertion of the vector values, the equation system is reduced to one component of the force-, length- and magnetic field vector:

$$
\left[\begin{array}{l}
d F_{d i s x} \\
d F_{d i s y} \\
d F_{d i s z}
\end{array}\right]=I\left(\begin{array}{c}
0 \\
0 \\
l_{x} B_{e x y}
\end{array}\right)
$$

The design for the coil with a C-shape is aligned with the anatomy of a slim thigh and is characterized by an air gap of $200 \mathrm{~mm}$ (Figure 5). The positioning of the coil around the thigh ensures the placement of the magnetic poles of the oscillators in the magnetic field. Hereby, a directed, reinforced and selective displacement of the single oscillator can be realized. The quadratic cross section of both coils of $10 \times 10 \mathrm{~mm}$ reduces the buckling of the magnetic field lines between both poles. 
Figure 5. Overview of the dimension of the core for the C-coil.

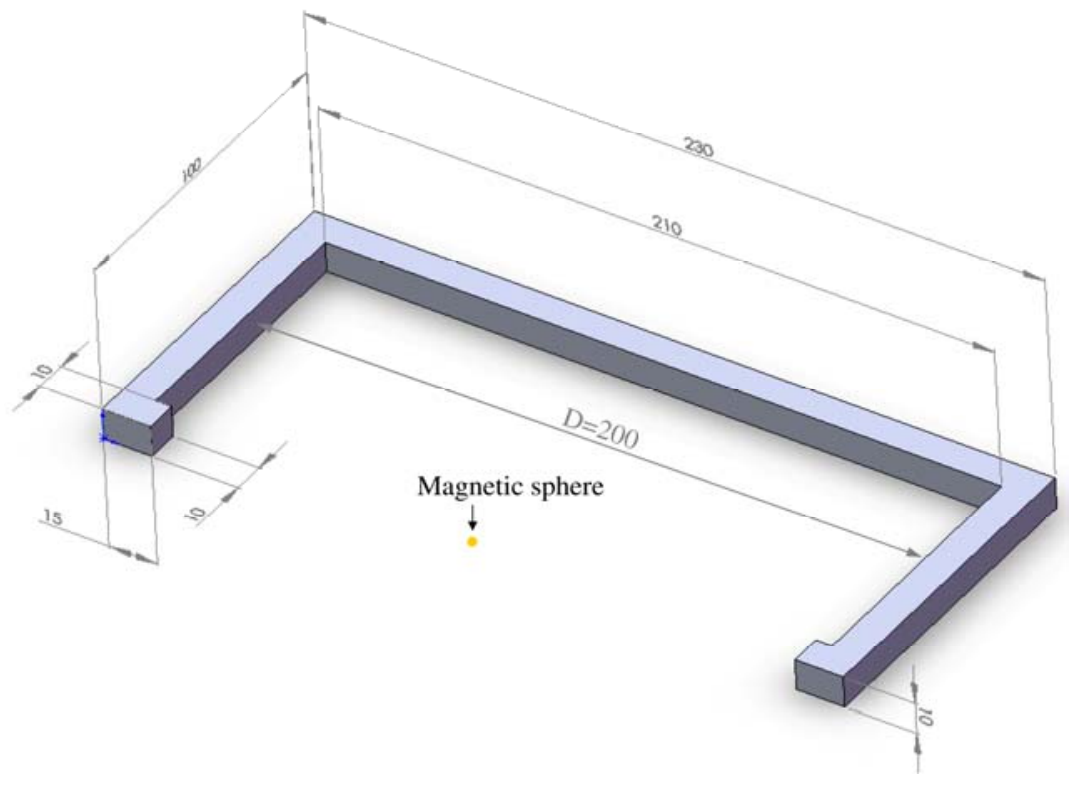

The simulation model was constructed with blanket planes in a distance of $0.125 \mathrm{~mm}$ to the coil core. This distance is equal to the manufacturing tolerance. The basic area of the blanket was assembled from the single areas of the cross sections of the coils multiplied with the number of the coils. In order to produce volume elements with equal volume along the surfaces of the coil core, the area of the equivalent areas was calculated. A volume-current density was assigned to the volume elements. Therefore, this ensured that the current I was consistent over the surface of the coil core. For the modelling of the oscillator, the magnetic sphere was simulated in the centre of the magnetic field. The magnetic sphere was placed at the distance $1 / 2 \mathrm{D}$ to both of the poles. For the dimensioning of the inductive unit, the magnitude of the magnetic field at the equator of the magnetic sphere is vitally important. The movement of a magnetic sphere in an alternating magnetic field affords the application of the solver for static frequency independent fields. The coil core is simulated with the properties of iron oxide $\left(\mathrm{Fe}_{2} \mathrm{O}_{3}\right)$, while for the magnetic sphere the material parameters for $\mathrm{NdFeB}$ are assigned (Table 2). For volume elements of the coils and for the surrounding of the system, a relative permeability of $\mu_{\mathrm{r}}=1$ is assumed.

Table 2. Overview of the material properties used for the simulation of the excitation coils.

\begin{tabular}{ccccc}
\hline Material & $\begin{array}{c}\text { Relative } \\
\text { Permeability } \boldsymbol{\mu}_{\mathbf{r}}\end{array}$ & $\begin{array}{c}\text { Magnetic } \\
\text { Remanence } \mathbf{B}_{\mathbf{r}}(\mathbf{T})\end{array}$ & $\begin{array}{c}\text { Coercive Field } \\
\text { Strength } \mathbf{H}_{\mathbf{C}}(\mathbf{A} / \mathbf{m})\end{array}$ & $\begin{array}{c}\text { Conductivity } \\
\boldsymbol{\varsigma}(\mathbf{S} / \mathbf{m})\end{array}$ \\
\hline $\mathrm{NdFeB}$ & - & 1.3 & $923,098.670$ & $833,333.330$ \\
$\mathrm{Fe}_{2} \mathrm{O}_{3}$ & 650 & 0.15 & 35 & $>1 \times 10^{-5}$ \\
\hline
\end{tabular}

Assuming a consistent distribution at the surface of the coil and A as the area created by the coil cross sections, current I is calculated:

$$
I=\frac{J A}{N}
$$


The entry surface of current I at every blanket face is defined using the right hand rule. The current I automatically flows in every volume element, whereas a consistent distribution of $I_{c}$ along the coil core at all sides of the geometry is ensured (Figure 6). With the same area and an increasing number of windings $\mathrm{N}$, it is assumed that every winding cross section decreases. Accordingly, the current linkage $\Theta$ increases, because a higher $N$ per area unit is flowed through by $I_{c}$.

Figure 6. Core of the C-coil with circular direction of the assigned current $\mathrm{I}_{\mathrm{c}}$.

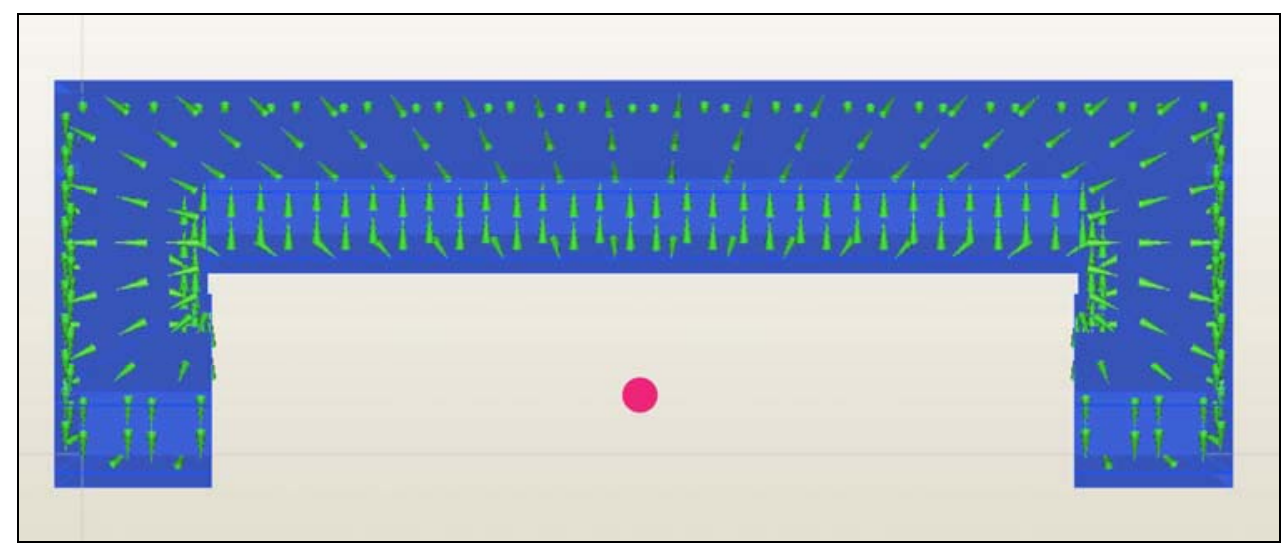

The direction of the magnetization $\mathrm{B}_{\mathrm{S}}$ of the magnetic sphere is defined in the $\mathrm{z}$-direction. During discretization, the volumes of the model geometry and single surfaces were meshed. For volume elements with assigned $I_{c}$, 3D-brick elements (hexahedral elements) were applied. Furthermore, all surfaces of the coil core and the magnetic sphere were meshed using 2D-triangle elements. The relevant parameters for the dimensioning of the coil are the magnetic flux density $B_{\text {ex }}$, especially in the $\mathrm{x}$-direction, the produced displacement force effect $\mathrm{F}_{\text {dis }}$, the moment which is developed around the $y$-axis of the magnetic sphere and the displacement of the conductor swing. The $y-$ and $z$-components of the magnetic field developed a force at the magnetic sphere. This force has no influence because of the directed displacement of the oscillator.

For determination of the optimal range, the coil number of the excitation coil was defined as $\mathrm{N}=2,000$. Following a convergence study, the components were meshed with approximately 9,000 elements. Validation of the numerical model was carried out by measuring $B_{\text {ex }}$ of the existing cylindrical coil using a teslameter (FM210 Teslameter, Projekt Elektronik GmbH, Berlin, Germany). Therefore, the sensor head was positioned in the center in front of the cylindrical coil. Hence, the distance between coil and sensor was varied in steps of $10 \mathrm{~mm}$ up to $60 \mathrm{~mm}$. In addition, the effective current through the coil was measured using a multimeter (Agilent Technologies U1242B, Santa Clara, CA, USA).

Different parameters for I and $\mathrm{N}$ were simulated to estimate a range in which the excitation of all oscillators could be guaranteed. In order to ensure a selective excitation of the individual oscillators, three different planes were evaluated. The chosen planes are equal to the position of the first three oscillators located distally in the hip stem. Plane 1 (reference plane) is defined with $\mathrm{z}=5 \mathrm{~mm}$, plane 2 with $\mathrm{z}=19.7 \mathrm{~mm}$ and plane 3 with $\mathrm{z}=36.5 \mathrm{~mm}$. A reference point in the middle of the air gap is assumed, where the magnetic flux density $B_{\text {ex }}$ can be compared. Therefore the quotients of plane 2 to plane 1 and plane 3 to plane 1 were calculated. 


\section{Results and Discussion}

\subsection{Parameter Analysis of the Internal System}

Within the eigenfrequency analysis of different oscillators, the highest peak of the resonance curve was determined (Figure 7). The labeled oscillator types in Table 3 were chosen for further measurements and as the basis for the design optimization using the Finite-Element-Method. To guarantee the long term stability, the geometry of the oscillators was chosen to be as small as possible, because additional gaps in the implant cannot be obviated using the proposed concept.

Figure 7. Resonance curve exemplarily for the oscillator type $4 \times 2 \times 0.05 \mathrm{~mm}$.

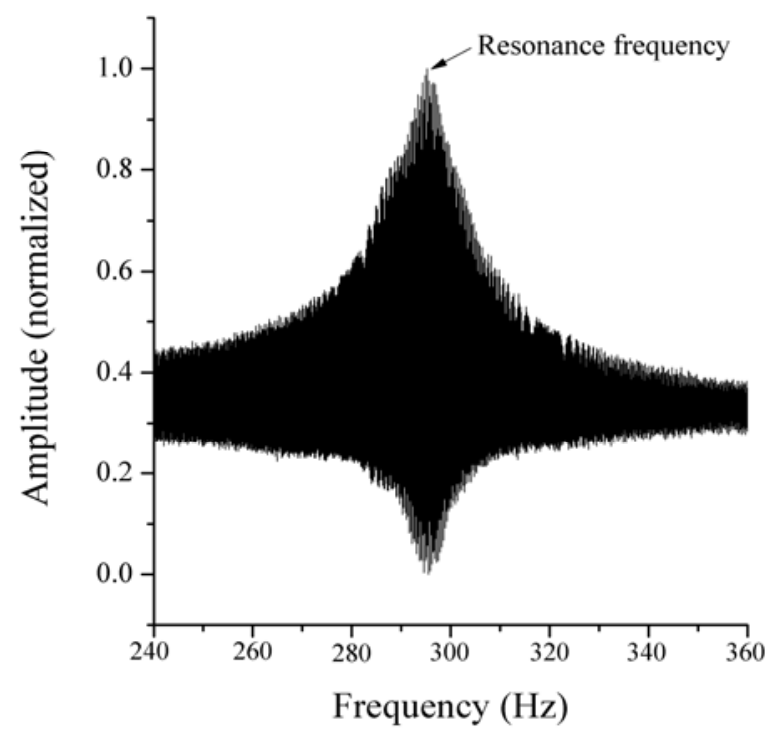

Table 3. Results of the eigenfrequency measurement as mean value (f) and associated standard deviation (sd) of different oscillators (bolded values are the parameters chosen for further investigations).

\begin{tabular}{|c|c|c|}
\hline Geometry (mm) & $\mathbf{f ( H z )}$ & $\mathbf{s d}(\mathbf{H z})$ \\
\hline $3 \times 2 \times 0.05$ & 367.00 & 3.65 \\
$\mathbf{4} \times \mathbf{2} \times \mathbf{0 . 0 5}$ & $\mathbf{2 8 5 . 6 2}$ & $\mathbf{0 . 8 8}$ \\
$5 \times 2 \times 0.05$ & 219.94 & 0.38 \\
$\mathbf{6} \times \mathbf{2} \times \mathbf{0 . 0 5}$ & $\mathbf{1 4 3 . 6 0}$ & $\mathbf{1 . 0 6}$ \\
$7 \times 2 \times 0.05$ & 124.16 & 0.20 \\
$3 \times 1 \times 0.05$ & 361.93 & 3.00 \\
$\mathbf{4} \times \mathbf{1} \times \mathbf{0 . 0 5}$ & $\mathbf{2 4 0 . 1 4}$ & $\mathbf{0 . 4 3}$ \\
$5 \times 1 \times 0.05$ & 122.57 & 1.19 \\
$6 \times 1 \times 0.05$ & 114.54 & 0.28 \\
$7 \times 1 \times 0.05$ & 71.68 & 0.33 \\
$7 \times 2 \times 0.10$ & 229.25 & 0.56 \\
\hline
\end{tabular}

Attaining an excitation of the oscillators with a length of $3 \mathrm{~mm}$ was difficult, due to the high energy expenditure, although the distance between coil and oscillator was only $30 \mathrm{~mm}$. Thus, oscillators which exhibit a length of $4 \mathrm{~mm}$ were chosen for the passive sensor array in the total hip stem. This was 
done to keep the gaps in the hip stem as small as possible. According to Equation (1) an increase of the thickness of the flat spring produces an extensive increase in the eigenfrequency of the oscillator. Unwanted oscillation signals of the proximate oscillator are small in the higher frequency range compared to a eigenfrequency below $200 \mathrm{~Hz}$. Therefore, both oscillators above $200 \mathrm{~Hz}$ need a smaller difference in the eigenfrequency than the oscillator with a lower eigenfrequency due to the lower energy input required.

Determination of the distance $d_{1}$ between the magnetic sphere and the membrane was a further aim of this study. $d_{1}$ must be ascertained because of the energy influence of the back-spring of the oscillator at the membrane [24]. For realization of the passive sensor array, distance $\mathrm{d}_{1}$ of $2.10 \mathrm{~mm}$ was chosen, as it supports the highest displacement of oscillator $144 \mathrm{~Hz}$. For oscillator $240 \mathrm{~Hz}$ and $286 \mathrm{~Hz}$ a distance of 2.05 was chosen (Table 4 ).

Table 4. Results of the measurement of the displacement $d_{2}$ as the highest amplitude to configure the optimal distance $\mathrm{d}_{1}$ between the centre of the magnetic sphere and the membrane (bolded values are the distances chosen for further investigations).

\begin{tabular}{|c|c|c|c|c|c|c|}
\hline \multirow{2}{*}{$\mathbf{d}_{\mathbf{1}}(\mathbf{m m})$} & \multicolumn{2}{|c|}{ Oscillator $\mathbf{1 4 4} \mathbf{~ H z}$} & \multicolumn{2}{c|}{ Oscillator $\mathbf{2 4 0} \mathbf{~ H z}$} & \multicolumn{2}{c|}{ Oscillator $\mathbf{2 8 6} \mathbf{~ H z}$} \\
\cline { 2 - 7 } & $\mathbf{d}_{\mathbf{2}}(\mathbf{m m})$ & $\mathbf{s d} \mathbf{( m m})$ & $\mathbf{d}_{\mathbf{2}}(\mathbf{m m})$ & $\mathbf{s d}(\mathbf{m m})$ & $\left.\mathbf{d}_{\mathbf{2}} \mathbf{( m m}\right)$ & $\mathbf{s d} \mathbf{( m m})$ \\
\hline 2.05 & 0.341 & 0.003 & $\mathbf{0 . 2 9 1}$ & $\mathbf{0 . 0 0 3}$ & $\mathbf{0 . 2 2 4}$ & $\mathbf{0 . 0 0 3}$ \\
2.10 & $\mathbf{0 . 7 2 1}$ & $\mathbf{0 . 0 0 5}$ & 0.271 & 0.003 & 0.215 & 0.003 \\
2.15 & 0.623 & 0.003 & 0.265 & 0.002 & 0.213 & 0.005 \\
2.20 & 0.568 & 0.001 & 0.231 & 0.003 & 0.161 & 0.003 \\
2.25 & 0.324 & 0.003 & 0.211 & 0.003 & 0.119 & 0.003 \\
\hline
\end{tabular}

The analysis of the influence of the cylindrical coil on the proximal oscillators showed a high influence on the proximal oscillator for the $144 \mathrm{~Hz}$ and the $286 \mathrm{~Hz}$ oscillator at distances between 5 and $20 \mathrm{~mm}$ (Table 5). This can be explained by the high energy input of the coil on the $286 \mathrm{~Hz}$ oscillator due to a forced oscillation in the higher frequency range of the $144 \mathrm{~Hz}$ oscillator. In all cases, it could be shown that the displacement of the proximate oscillator is smaller than the distance between sphere and the membrane $(2.1 \mathrm{~mm})$, except for oscillator configuration $144 \mathrm{~Hz}$ and $286 \mathrm{~Hz}$. For this reason, a distance $d_{3}$ of $20 \mathrm{~mm}$ between every magnetic sphere was chosen to reduce the influence of the magnetic field lines as much as possible.

Table 5. Results of the measurement of the displacement $d_{2}$ of the influenced oscillator proximal to the excited oscillator at different distances $d_{3}$.

\begin{tabular}{|c|c|c|c|c|c|c|}
\hline \multirow{2}{*}{$\mathbf{d}_{\mathbf{3}}(\mathbf{m m})$} & \multicolumn{2}{|c|}{$\mathbf{1 4 4} \mathbf{~ H z}-\mathbf{2 4 0} \mathbf{~ H z}$} & \multicolumn{2}{c|}{$\mathbf{1 4 4} \mathbf{~ H z}-\mathbf{2 8 6} \mathbf{~ H z}$} & \multicolumn{2}{c|}{$\mathbf{2 4 0} \mathbf{~ H z}-\mathbf{2 8 6} \mathbf{~ H z}$} \\
\cline { 2 - 7 } & $\left.\mathbf{d}_{\mathbf{2}} \mathbf{( m m}\right)$ & $\mathbf{s d} \mathbf{( m m})$ & $\left.\mathbf{d}_{\mathbf{2}} \mathbf{( m m}\right)$ & $\mathbf{s d} \mathbf{( m m})$ & $\left.\mathbf{d}_{\mathbf{2}} \mathbf{( m m}\right)$ & $\mathbf{s d} \mathbf{( m m})$ \\
\hline 5 & 0.196 & 0.003 & 1.05 & 0.005 & 0.146 & 0.001 \\
10 & 0.123 & 0.002 & 0.865 & 0.001 & 0.132 & 0.002 \\
20 & 0.072 & 0.003 & 0.243 & 0.003 & 0.113 & 0.001 \\
30 & 0.057 & 0.003 & 0.031 & 0.001 & 0.094 & 0.002 \\
40 & 0.032 & 0.001 & 0.015 & 0.002 & 0.075 & 0.002 \\
60 & 0.012 & 0.001 & 0.002 & 0.001 & 0.054 & 0.001 \\
\hline
\end{tabular}


Based on these results, the design optimization of the oscillators for application in a total hip stem was performed using the Finite-element-method (Figure 8). A challenge of the application of oscillators as a passive sensor array is the significant effort required to determine the exact eigenfrequency of every oscillator after the manufacturing process for precise excitation. A further limitation is the substantial effort required to measure the back coupled acoustic signal at every membrane to create a baseline measurement. Further work therefore will include the investigation of the long-term stability of the oscillators.

Figure 8. Results of the simulated oscillators creating the geometry variations.
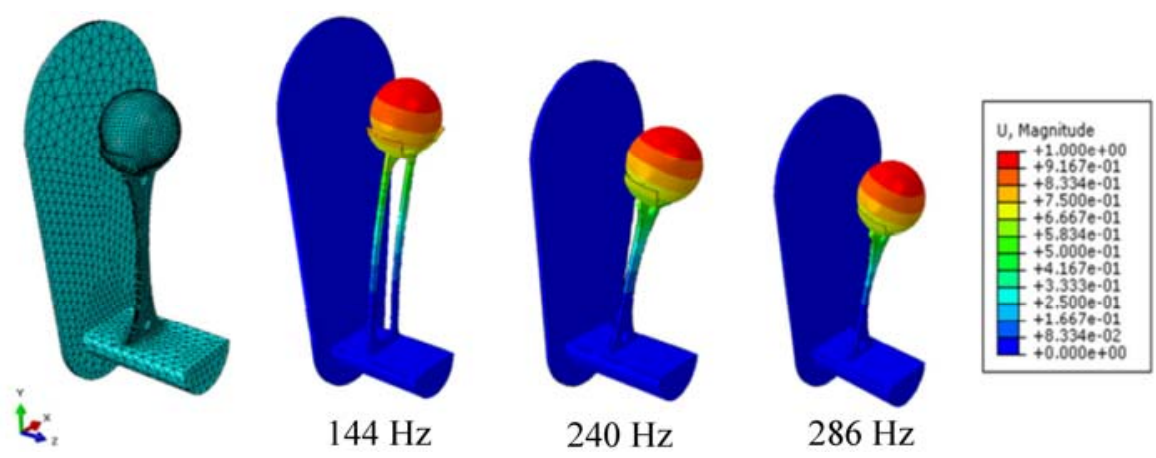

For the first arrangement of the passive sensor array inside a THR, a custom straight hip stem (Mathys CBH shaft standard, Mathys, Bettlach, Switzerland) was used (Figure 9). Four oscillators were positioned at the lateral face due to an accentuated fixation and bone ingrowths at the distal lateral part of this stem type as a first demonstrator [25]. Hence, distal loosening has a high influence on the stability of the hip stem. The optimized design of the oscillator geometry is manufactured by a generative procedure with rapid prototyping. Here, the oscillators are constructed with membrane as one component (Figure 9).

Figure 9. Sensor-equipped demonstrator: Arrangement of the passive sensor array inside a total hip stem.

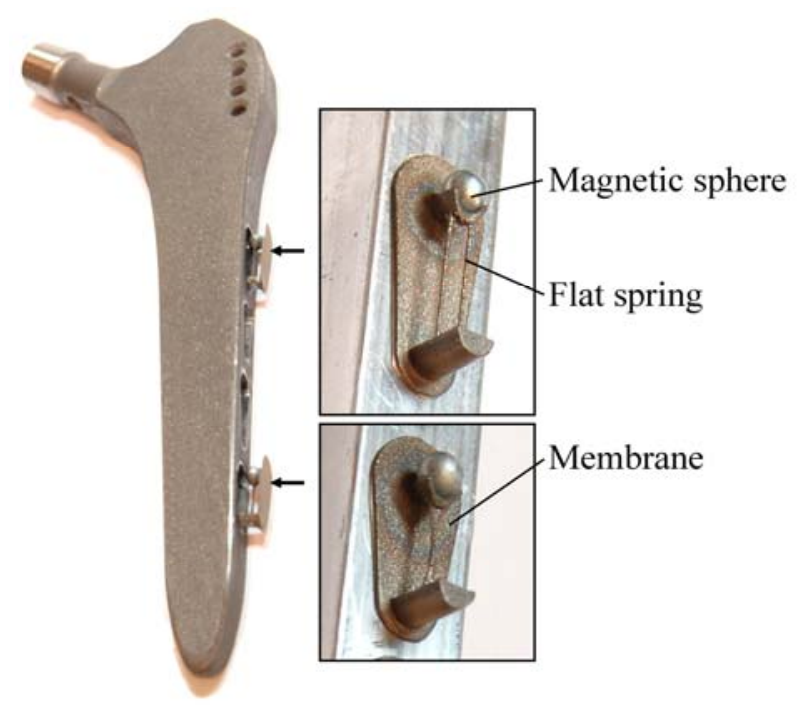


In order to realize a first test, the demonstrator was proximally fixed in a clamping device and the oscillators were excited with their eigenfrequency and hence recorded by an accelerometer (KS95B, Metra, Radebeul, Germany) positioned at the middle of the total hip stem. The selective excitation of each oscillator could be proven. Furthermore, the frequency spectrum was calculated with a resolution of $0.6 \mathrm{~Hz}$ using Fast Fourier transformation (FFT) with a Hanning window of the time signal. The recorded structure borne sound signal consists of many different frequencies, which compose the complete sound spectrum. For this reason, the highest peak of the normalized two-dimensional magnitude-frequency-plot was analyzed. The frequency spectrum shows a difference between the excitation of the different oscillators (Figure 10). For example, in the lower frequency area a difference of $219 \mathrm{~Hz}$ could be detected. On the one hand this can be explained by the measurement location, on the other hand, the membrane of the oscillator type $240 \mathrm{~Hz}$ is smaller compared to type $144 \mathrm{~Hz}$. Due to the differences in the structure-borne-sound signal, this first measurement applying the new approach has a high potential to detect and locate implant loosening in a total hip stem.

Figure 10. Frequency spectrum of the measurement using the demonstrator and exciting the oscillator type $144 \mathrm{~Hz}$ and type $240 \mathrm{~Hz}$.

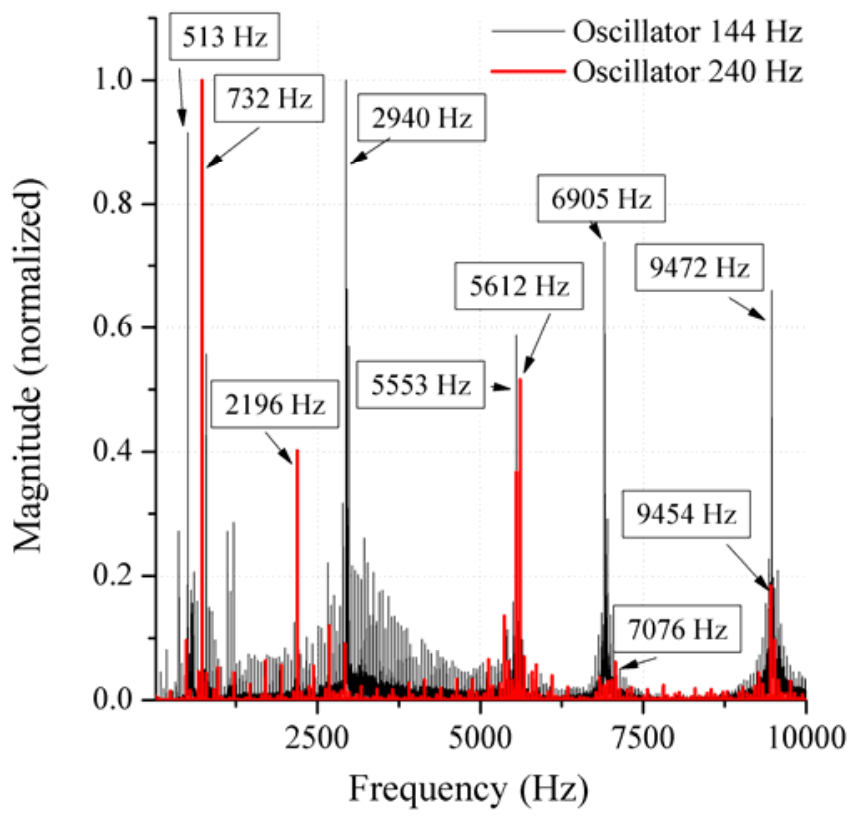

\subsection{Results of the Design Analysis of the Extracorporeal System}

The results of the measured and simulated data of the cylindrical coil revealed an acceptable compliance (Table 6). A resulting maximum deviation of $7.2 \%$ can be considered a valid basis for simulation of the C-coil.

Table 6. Results of the validation of the numerical model of the cylindrical coil using an effective current of $2.24 \mathrm{~A}$.

\begin{tabular}{|c|c|c|c|c|c|c|}
\cline { 2 - 7 } \multicolumn{1}{c|}{} & \multicolumn{6}{c|}{$\mathbf{B}_{\text {ex }}(\mathbf{m T})$} \\
\hline Distance to coil & $\mathbf{1 0} \mathbf{~} \mathbf{m m}$ & $\mathbf{2 0} \mathbf{~} \mathbf{m m}$ & $\mathbf{3 0} \mathbf{~ m m}$ & $\mathbf{4 0} \mathbf{~} \mathbf{m}$ & $\mathbf{5 0} \mathbf{~ m m}$ & $\mathbf{6 0} \mathbf{~ m m}$ \\
\hline Experiment & 60.1 & 30.2 & 19.1 & 12.8 & 8.8 & 6.3 \\
Simulation & 55.8 & 30.3 & 19.5 & 12.4 & 8.5 & 6.5 \\
\hline
\end{tabular}


The nominal values of the magnetic flux densities $B_{\text {ex }}$ were evaluated. These could be determined by assessing a measurement path along the $\mathrm{x}$-axis (Figure 11). The magnetic field distribution between both poles starts at $\mathrm{x}=15 \mathrm{~mm}$ and ends at $\mathrm{x}=215 \mathrm{~mm}$. The poles are located at $\mathrm{x}=0$ to $\mathrm{x}=15 \mathrm{~mm}$ and at $x=215 \mathrm{~mm}$ to $x=230 \mathrm{~mm}$. The centre of the magnetic sphere is located at $\mathrm{x}=115 \mathrm{~mm}$.

Figure 11. Contour plot of the cylindrical coil (left) and the C-coil (right) with three evaluated planes. Illustration of the measurement path for evaluation of $\mathrm{B}_{\mathrm{ex}}$ along the $\mathrm{x}$-axis.

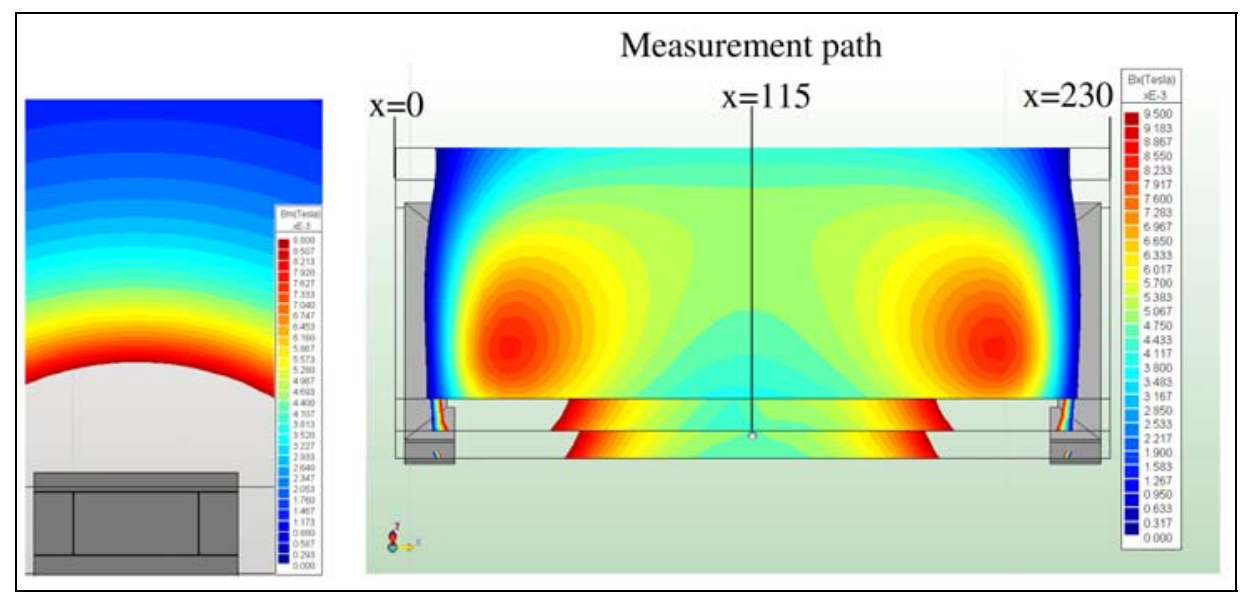

Directly at the poles, a high magnetic flux density of $\mathrm{B}_{\mathrm{ex}}=240 \mathrm{mT}$ could be identified. A strong decrease of $B_{\mathrm{ex}}$ could be visualized at the changeover of the ferrite core to air. For the cylindrical coil, a magnetic flux density of $B_{\text {exCyl }}=1.27 \mathrm{mT}$ over an operating distance of $100 \mathrm{~mm}$ (consistent to $\mathrm{x}=115 \mathrm{~mm}$ ) was calculated (Figure 11). This corresponds to one half of the magnetic flux density, which is necessary for the displacement of $144 \mathrm{~Hz}$ oscillator type. In contrast to the cylindrical coil, the C-coil achieves a magnetic flux density of $\mathrm{B}_{\mathrm{ex}}=1.79 \mathrm{mT}$, applying $\mathrm{N}=1,000$ and $\mathrm{I}_{\mathrm{c}}=2.24 \mathrm{~A}$ (nominal current $\mathrm{I}_{\mathrm{N}}=3 \mathrm{~A}$ ). One reason for the lower $\mathrm{B}_{\mathrm{exCyl}}$ is the absence of the directional characteristics of the cylindrical coil, which is achieved by the $\mathrm{C}$-coil with opposed poles. Excitation of the oscillators using the cylindrical coil can only be realized in the near field at the poles, because the field diverged with increasing distance and lost intensity. It should be mentioned that the external field components are not considered during the transfer of the magnetic sphere into the conductor swing. These components have an influence on the virtual current I which is developed at the surface of the magnetic sphere, producing an increase. Hence, the magnetic flux density that is used for the displacement of the oscillator decreases. In this case, a conservative consideration of the magnetic field is given. Furthermore, the hexahedral profile of the C-coil compared to the cylindrical coil is apparent, which can be explained by the directional characteristics and the more homogeneous distribution of the magnetic field lines between both poles.

Based on these results, different parameters of $\mathrm{N}$ and $\mathrm{I}_{\mathrm{c}}$ were simulated in order to get the range over which all oscillator types can be excited (Figure 12). Due to better directional characteristics of the $\mathrm{C}$-coil, it is possible to produce an adequate magnetic flux density with relatively small currents of $I_{c}=2.5 \mathrm{~A}$ in the centre of the air gap. The limitation of $I_{c}$ to $3.0 \mathrm{~A}$ referred to the application in an electric circuit, in which the excitation coil has to be integrated. Important values of $B_{\mathrm{ex}}$ between $\mathrm{x}=80 \mathrm{~mm}$ and $\mathrm{x}=150 \mathrm{~mm}$ are relevant for the desired displacement force of the oscillators. Within this range $B_{\text {ex }}$ lies between $2.42 \mathrm{mT}$ and $10.1 \mathrm{mT}$. The numerical values of $B_{\text {ex }}$ ensure a displacement 
of the $240 \mathrm{~Hz}$ and $144 \mathrm{~Hz}$ oscillator types inside the air gap simulating $\mathrm{N}=3,000$ and a current of $\mathrm{I}_{\mathrm{c}}=2.0 \mathrm{~A}$. Figure 12 shows that the $286 \mathrm{~Hz}$ oscillator type can only be displaced by a C-coil configured with $\mathrm{N}=5,000$ and $\mathrm{I}_{\mathrm{c}}=2.5 \mathrm{~A}$. The numerical values of $\mathrm{B}_{\mathrm{ex}}$ are determined for the equator of the magnetic sphere. In case of a higher permeability, the relations do not change significantly.

Figure 12. Comparison of the generated magnetic flux densities of the C-coil using different windings $\mathrm{N}$ and currents $\mathrm{I}_{\mathrm{c}}$ in comparison with the calculated values of the different oscillator types.

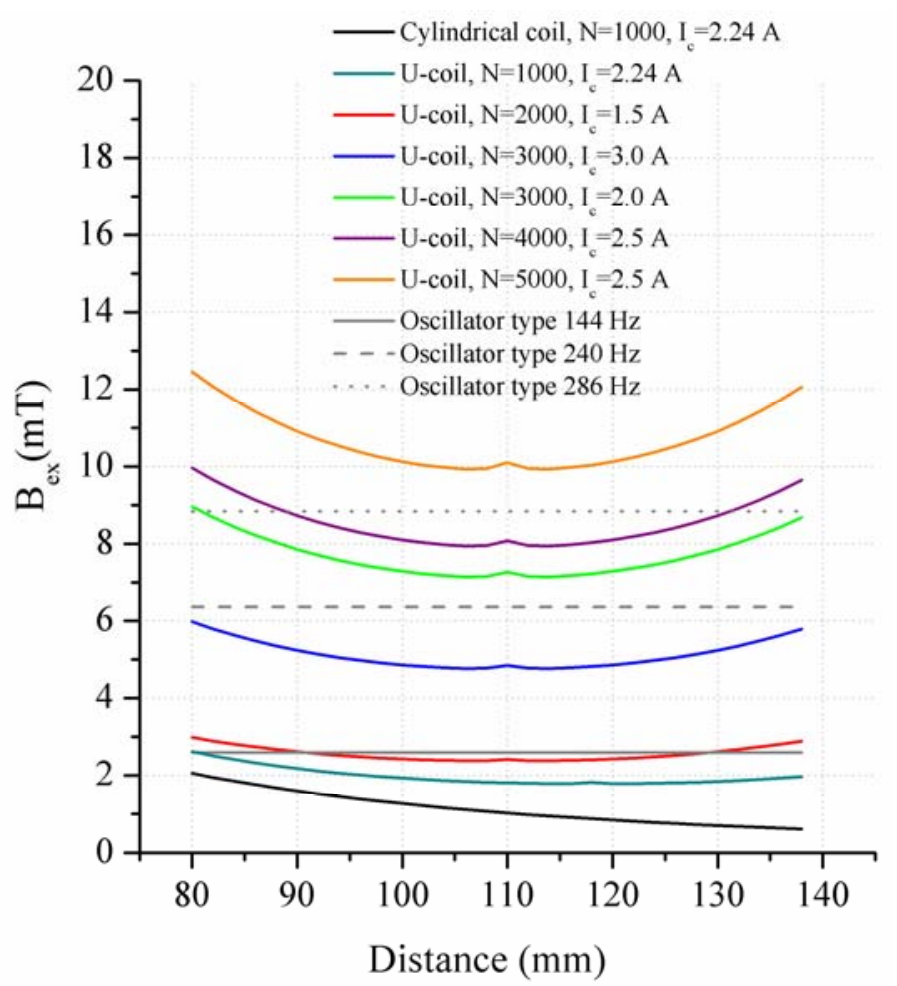

Figure 13. Contour plot of the distribution of $B_{e x}$ in three different planes of the magnetic spheres using a winding number of $\mathrm{N}=1,000$ and a current $\mathrm{I}_{\mathrm{c}}=2.24 \mathrm{~A}$.

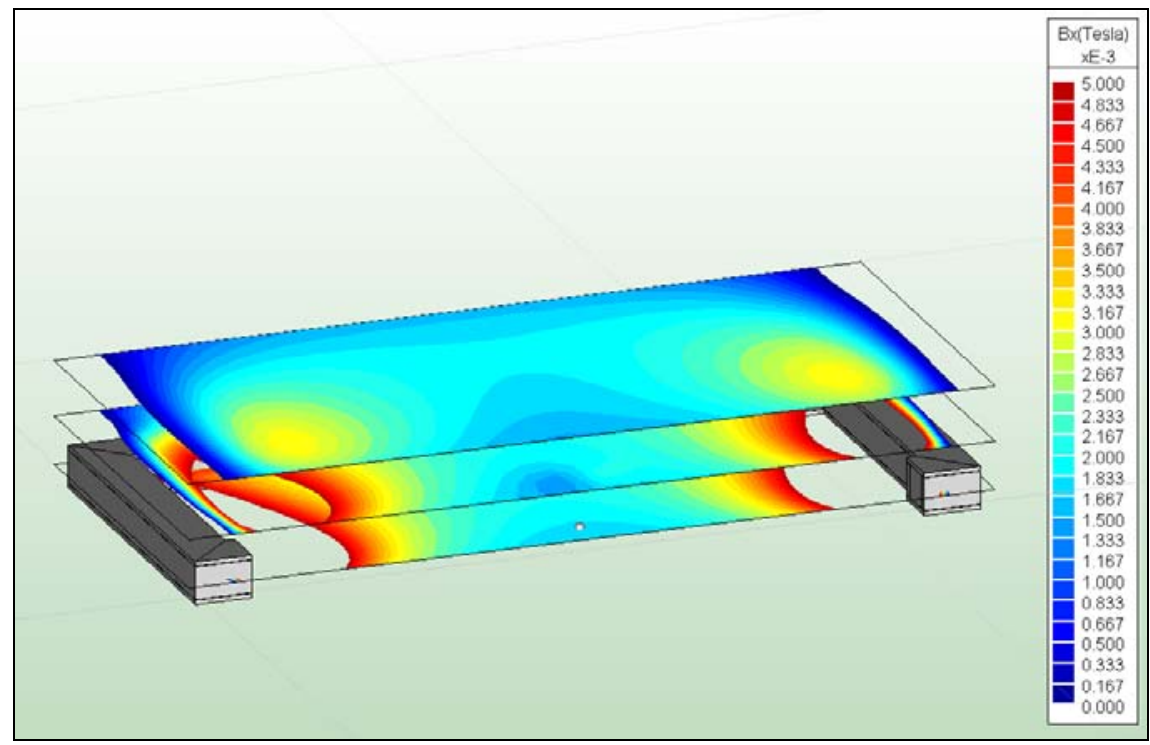


For the estimation of the selectivity, plane 2 and plane 3 are compared to plane 1 (Figure 13, Table 7). It could be shown that $B_{\text {ex }}$ of plane 1 to plane 3 is attenuated by $13 \%$. Upon comparison of plane 1 and plane 2, an attenuation of the intensity of 5\% was carried out. For the realization of an optimized excitation coil, the magnetic field was charged with a security factor of 0.06 , without losing the advantage of a selective excitation.

Table 7. Results of the magnetic flux density in the center of the air gap using different parameters.

\begin{tabular}{|c|c|c|c|c|}
\hline $\mathbf{N}$ & $\mathbf{I}_{\mathbf{c}}(\mathbf{A})$ & $\mathbf{B}_{\text {ex }}$ Plane 1 $(\mathbf{m T})$ & $\mathbf{B}_{\text {ex }}$ Plane 2 $(\mathbf{m T})$ & B $_{\text {ex }}$ Plane 3 $(\mathbf{m T})$ \\
\hline 2,000 & 1.5 & 2.42 & 2.31 & 2.12 \\
& 2.0 & 4.85 & 4.62 & 4.23 \\
3,000 & 3.0 & 7.27 & 6.94 & 6.35 \\
& 2.5 & 8.08 & 7.71 & 7.05 \\
4,000 & 2.5 & 10.10 & 9.63 & 8.82 \\
5,000 &
\end{tabular}

Despite an increase of $6 \%$ in plane $1, \mathrm{~B}_{\mathrm{ex}}$ in plane 3 is below the displacement of the oscillator of the desired magnetic field. The selectivity can be reached by a variation of the excitation frequency, and is ensured for the excitation in plane 1, 2 and 3 (Figure 13). In contrast to the cylindrical coil, the $\mathrm{C}$-coil allows the displacement of the oscillators over a distance of $100 \mathrm{~mm}$. Using the cylindrical coil over this distance, an excitation is not possible. This means that with the C-coil a magnetic flux density of $\mathrm{B}_{\mathrm{ex}}$ can be generated, which is $719 \%$ of $\mathrm{B}_{\mathrm{ex}}$ of the cylindrical coil. The influence of the air gap on $\mathrm{B}_{\mathrm{ex}}$ is obvious, and the magnetic field lines describe a curve between both poles with decreasing gap size. This is a disadvantage for the positioning of the coil at the patient's leg and hence for the selectivity of the oscillators. A further aspect under consideration is the cross section of the simulated coil. A shape with circular cross section is always the best choice regarding a loss of magnetization. Due to the manufacturing process using a ferrite core, the coil could only be realized with a hexahedral cross section.

\subsection{Benefits}

Over time, several approaches for diagnosing loosening to total hip replacements have been investigated. It was shown that mechanical oscillations of the implant-bone-system could be excited with an electrodynamic shaker [12,17-19]. A challenge which cannot be neglected is the influence of the shaker on the patient's knee, due to the manifestation of pain and a defined contact between shaker and skin. Application of the proposed passive sensor array will not be painful, because of the low oscillator mass and small excitation frequencies. Nevertheless, electronic devices using telemetry and accelerometers that were inserted in the total hip stem in former studies did not withstand the standard sterilization process $[21,23]$. The simple and robust passive sensor array has a high resistance to sterilization and impaction during implantation. Due to the integration of several oscillators along the total hip stem, the presented approach is more sensitive to implant loosening, because locations of implant loosening can be diagnosed from the read-out of different sounds produced by the impingement of the oscillators at the membranes. This was not possible using former approaches. 
Hao et al. presented a further concept that included a measurement system for investigation of implant migration and micro movements [26]. This is realized with two coils that are coupled over a core. During the implantation; the core is in contact with the total hip stem in order to detect a measurable voltage. For this concept; an active electronic device is inserted in the femur. The way that the system is implanted is significant; since imprecise measurements during positioning cannot be compensated for by the calibration process [26]. In comparison; the proposed passive sensor array is independent from the implantation process of the total hip stem. Furthermore; the sensor array only needs one postoperative baseline measurement.

\subsection{Limitations}

Due to the integration of the passive sensor array at the lateral side of the hip stem, the long-term stability of the total hip stem was considered in Finite-Element-Analyses. Unpublished data about maximum stresses and static strength show promising results. A static analysis showed that stresses were in an acceptable range if the cavities for the oscillator-membrane-component are small enough. The feasibility has to be proved by a long-term fatigue strength test. Thus, the oscillators may need to be further miniaturized, taking into consideration the production of a reasonable acoustic signal, which should be strong enough to be detected by a microphone or at the skin by an accelerometer. Further miniaturization must be considered, although the manufacturing process is limited due to the thickness of the membrane and the spring. Thus, the eigenfrequency of the oscillator increases and hence skin effects in the coil can appear. This effect results in higher currents, which cannot be produced to displace the oscillators. Another point for consideration is the measurement system, which has to be adjusted to the resonant eigenfrequencies of the oscillators. The functionality of acoustic sound and structural vibration detection was demonstrated on overdimensioned models [24] and in in vitro studies using porcine forelegs $[27,28]$. Further work requires the determination of the sensitivity of the passive sensor array to implant loosening.

Furthermore, an additional stand for the coil to achieve a precise excitation should be considered. This could be achieved in further steps by varying the magnetic field strength of the magnetic spheres in order to determine the location of each individual oscillator by applying sensors which are capable of measuring the magnetization at every location.

\section{Conclusions}

In the present study, a novel passive sensor array was investigated for implementation in endoprosthetic implants in order to identify implant loosening. The main focus of the work was to achieve a reproducible and selective excitation of sound waves inside the implant. A basic sensor array was constructed and tested for integration in the total hip stem. Furthermore, eigenfrequencies of the oscillators were determined to identify three oscillator types with a high difference in order to ensure the excitation selectivity. Optimization of the inductive extracorporeal unit of the measurement system using finite-boundary simulation for a cylindrical and C-shaped coil revealed the highest selectivity by exciting the oscillator using a $\mathrm{C}$-shape. The presented simulations showed how the coils behave during application and how the magnetic field characteristics are developed. With the comparison in the different planes and the resulting attenuation of the intensity of the magnetic field, an appraisal of the 
selective excitation of the different oscillator types is possible. The results show that due to the variation of the oscillator geometry, the oscillators can be excited selectively. The parameters for the C-coil have to be chosen very precisely.

Based on these investigations, the passive sensor system reveals the potential for detection of implant loosening. The next steps include the further miniaturization of the oscillators and tests of functionality of the demonstrator. Furthermore measurements to determine the sensitivity of the proposed sensor system will be realized including in vivo studies and in clinical setting.

\section{Acknowledgments}

This research project was funded in part by the Deutsche Forschungsgemeinschaft (DFG - German Research Foundation) under Reg. No. KL 2327/1 and in part by the Ministry of Education, Science and Culture of the Federal State Mecklenburg-Vorpommern and by the European Union (VentureCup MV, FKZ: UR 10020).

\section{References}

1. Malchau, H.; Herberts, P.; Eisler, T.; Garellick, G.; Soderman, P. The Swedish total hip replacement register. J. Bone Jt. Surg. Am. 2002, 84-A, 2-20.

2. Cuckler, J.M. Unexplained pain after THR: What should I do? Orthopedics 2010, 33, 648.

3. Temmermann, O.P.; Raijmakers, P.G.; Berkhof, J.; David, E.F.; Pijpers, R.; Molenaar, M.A.; Hoekstra, O.S.; Teule, G.J.; Heyligers, I.C. Diagnostic accuracy and interobserver variability of plain radiography, subtraction arthrography, nuclear arthrography, and bone scintigraphy in the assessment of aseptic femoral component loosening. Arch. Orthop. Trauma Surg. 2006, 126, 316-323.

4. Zhang, Y.; Putnam, A.W.; Heiner, A.D.; Callaghan, J.J.; Brown, T. Reliability of detecting prosthesis/cement interface radiolucencies in total hip arthroplasty. J. Orthop. Res. 2002, 20, 683-687.

5. Huang, H.M.; Chiu, C.L.; Yeh, C.Y.; Lin, C.T.; Lin, L.H.; Lee, S.Y. Early detection of implant healing process using resonance frequency analysis. Clin. Oral Implants Res. 2003, 14, 437-443.

6. Glauser, R.; Sennerby, L.; Meredith, N.; Rée, A.; Lundgren, A.K.; Gottlow, J.; Hämmerle, C.H.F. Resonance frequency analysis of implants subjected to immediate or early functional occlusal loading-Successful vs. failing implants. Clin. Oral Implants Res. 2003, 15, 428-434.

7. Wang, S.; Liu, G.R.; Hoang, K.C.; Guo, Y. Identifiable range of osseointegration of dental implants through resonance frequency analysis. Med. Eng. Phys. 2010, 32, 1094-1106.

8. Pattijn, V.; Van Lierde, C.; Van der Perre, G.; Naert, I.; Vander Sloten, J. The resonance frequencies and mode shapes of dental implants: Rigid body behaviour versus bending behaviour. A numerical approach. J. Biomech. 2006, 39, 939-947.

9. Pastrav, L.C.; Jaecques, S.V.N.; Jonkers, I.; van der Perre, G.; Mulier, M. In vivo evaluation of a vibration analysis technique for the peri-operative monitoring of the fixation of hip prostheses. J. Orthop. Surg. Res. 2009, 4, 1-10.

10. Cristofolini, L.; Varini, E.; Pelgreffi, I.; Cappello, A.; Toni, A. Device to measure intra-operatively the primary stability of cementless hip stems. Med. Eng. Phys. 2006, 28, 475-482. 
11. Lannocca, M.; Varini, E.; Cappello, A.; Cristofolini, L.; Bialoblocka, E. Intra-operative evaluation of cementless hip implant stability: A prototype device based on vibration analysis. Med. Eng. Phys. 2007, 29, 886-894.

12. Rowlands, A.; Duck, F.A.; Cunningham, J.L. Bone vibration measurement using ultrasound: Application to detection of hip prosthesis loosening. Med. Eng. Phys. 2008, 30, 278-284.

13. Meredith, N.; Alleyne, D.; Cawley, P. Quantitative determination of the stability of the implant-tissue interface using resonance frequency analysis. Clin. Oral Implants Res. 1996, 7, 261-267.

14. Meredith, N.; Shagaldi, F.; Alleyne, D.; Sennerby, L.; Cawley, P. The application of resonance frequency measurements to study the stability of titanium implants during healing in the rabbit tibia. Clin. Oral Implants Res. 1997, 8, 234-243.

15. Koh, J.-W.; Yang, J.-H.; Han, J.-S.; Lee, J.-B.; Kim, S.-H. Biomechanical evaluation of dental implants with different surfaces: Removal torque and resonance frequency analysis in rabbits. J. Adv. Prosthodont. 2009, 1, 107-112.

16. Park, I.P.; Kim, S.K.; Lee, S.J.; Lee, J.H. The relationship between initial implant stability quotient values and bone-to-implant contact ratio in the rabbit tibia. J. Adv. Prosthodont. 2011, 3, 76-80.

17. Rosenstein, A.D.; McCoy, G.F.; Bulstrode, C.J.; McLardy-Smith, P.D.; Cunningham, J.L.; Turner-Smith, A.R. The differentiation of loose and secure femoral implants in total hip replacement using vibrational technique-An anatomical and pilot clinical study. Proc. Inst. Mech. Eng. 1989, 203, 77-81.

18. Li, P.L.S.; Jones, N.B.; Gregg, P.J. Vibration analysis in the detection of total hip prosthetic loosening. Med. Eng. Phys. 1996, 18, 556-600.

19. Georgiou, A.P.; Cunningham, J.L. Accurate diagnosis of hip prosthesis loosening using a vibrational technique. Clin. Biomech. 2001, 16, 315-323.

20. Qi, G.; Mouchon, W.P.; Tan, T.E. How much can a vibrational diagnostic tool reveal in total hip arthroplasty loosening. Clin. Biomech. 2003, 18, 444-458.

21. Puers, R.; Catrysse, M.; Vandevoorde, G.; Collier, R.J.; Louridas, E.; Burny, F.; Donkerwolcke, M.; Moulart, F. A telemetry system for the detection of hip prosthesis loosening by vibration analysis. Sens. Actuators A Phys. 2000, 85, 42-47.

22. Rieger, P. Implantable Measurement Systems with Wireless Digital Information Transmission. Ph.D. Thesis, Department of Electrical Engineering, University of Kaiserslautern, Kaiserslautern, Germany, 2 February 2001.

23. Marschner, U.; Grätz, H.; Jettkant, B.; Ruwisch, D.; Woldt, G.; Fischer, W.J.; Clasbrummel, B. Integration of a wireless lock-in measurement of hip prosthesis vibrations for loosening detection. Sens. Actuators A Phys. 2009, 156, 145-154.

24. Ruther, C.; Ewald, H.; Mittelmeier, W.; Fritsche, A.; Bader, R.; Kluess, D. A new concept for non-invasive radiation-free detection of implant loosening. J. Biomech. Eng. 2011, 133, 104503.

25. Draenert, K. Draenert, Y. Forschung und Fortbildung in der Chirurgie des Bewegungsapparates 3: Strain-Adaptive Bone Remodelling, 1st ed.; Art and Science München: München, Germany, 1992; pp. 49-56. 
26. Hao, S.; Taylor, J.T.; Bowen, C.R.; Gheduzzi, S.; Miles, A. Sensing methodology for in vivo stability evaluation of total hip and knee arthroplasty. Sens. Actuators A Phys. 2010, 157, 150-160.

27. Ruther, C.; Timm, U.; Ewald, H.; Mittelmeier, W.; Bader, R.; Kluess, D. Detecting sound vibration for the monitoring of implant loosening. In Proceedings of the 18th Congress of the European Society of Biomechanics, Lisbon, Portugal, 1-4 July 2012.

28. Ruther, C.; Timm, U.; Ewald, H.; Mittelmeier, W.; Bader, R.; Schmelter, R.; Lohrengel, A.; Kluess, D. Current possibilities for detection of loosening of total hip replacements and how intelligent implants could improve diagnostic accuracy. In Recent Advances in Arthroplasty, 1st ed.; Fokter, S.K., Ed.; InTech: Rijeka, Croatia, 2012; pp. 363-386.

(C) 2013 by the authors; licensee MDPI, Basel, Switzerland. This article is an open access article distributed under the terms and conditions of the Creative Commons Attribution license (http://creativecommons.org/licenses/by/3.0/). 